\title{
Measure of tripartite entanglement in bosonic and fermionic systems
}

\author{
Fabrizio Buscemi ${ }^{1,2, *}$ and Paolo Bordone ${ }^{3,4}$ \\ ${ }^{1}$ Dipartimento di Elettronica, Informatica, e Sistemi, Università di Bologna, Viale Risorgimento 2, I-40136 Bologna, Italy \\ ${ }^{2}$ ARCES, Alma Mater Studiorum, Università di Bologna, Via Toffano 2/2, I-40125 Bologna, Italy \\ ${ }^{3}$ Dipartimento di Fisica, Università di Modena e Reggio Emilia, I-41125 Modena, Italy \\ ${ }^{4}$ Centro S3, CNR-Istituto di Nanoscienze, Via Campi 213/A, I-41125 Modena, Italy
}

(Received 20 May 2011; published 2 August 2011)

\begin{abstract}
We describe an efficient theoretical criterion suitable for the evaluation of the tripartite entanglement of any mixed three-boson or three-fermion state, based on the notion of the entanglement of particles for bipartite systems of identical particles. Our approach allows one to quantify the accessible number of quantum correlations in the systems without any violation of the local particle number superselection rule. A generalization of the tripartite negativity is here applied to some correlated systems including the continuous-time quantum walks of identical particles (for both bosons and fermions) and compared with other criteria recently proposed in the literature. Our results show the dependence of the entanglement dynamics upon the quantum statistics: The bosonic bunching results in a low number of quantum correlations while Fermi-Dirac statistics allows for higher values of the entanglement.
\end{abstract}

DOI: 10.1103/PhysRevA.84.022303

PACS number(s): 03.67.Mn, 05.40.-a, 03.65.Ud

\section{INTRODUCTION}

The notion of the entanglement in bosonic or fermionic systems has been the subject of many recent discussions and controversies since it raises some conceptual questions about the nature of the quantum correlations appearing in such systems [1-5]. The main difficulty appearing in the definition of a criterion apt to classify and quantify the number of quantum correlations is due to the intrinsic indistinguishability of the particles supposed to be entangled, which results in the symmetrization or antisymmetrization of the wave function. This stimulated great interest in the scientific community and led to various proposals of entanglement criteria for identical particles. In particular, a number of works present in the literature have been focused on the analysis of bipartite entanglement $[1,3,4,6]$. In the paper by Schliemann et al. [1], the number of quantum correlations between two fermions is evaluated by considering the fermionic analog of the Schmidt rank, namely the Slater rank, which is given by the number of Slater determinants needed to expand the entangled states. The Schliemman criterion has been used to evaluate the entanglement in a number of bipartite systems of physical interest [7-9]; still it is discussed since it does not seem to behave correctly under one-site (local) and two-site (nonlocal) transformations [2]. In the approach developed by Zanardi [3], the entanglement is evaluated in terms of the quantum correlations between modes by mapping the Fock space of the modes themselves into qubit states. It has been shown that such a criterion overestimates the entanglement between the two parties of the quantum system since it conflicts with the local number particle superselection rule [4]. In order not to violate the latter, Wiseman and Vaccaro [4] have proposed an operational criterion for bipartite entanglement of identical particles. They define as entanglement of particles the maximum number of quantum correlations which can be extracted by means of local operation on the modes

\footnotetext{
*fabrizio.buscemi@unimore.it
}

from the two parties of the system and then set in standard quantum registers (composed of distinguishable qubits) $[4,6]$. Only recently a coherent picture on the entanglement of indistinguishable particles somehow including all the above criteria has been presented in the literature [5].

Though the entanglement of multipartite systems suggests more possibilities and phenomena with respect to the bipartite case, so far it has not been widely investigated. Its quantification would undoubtedly represent a key ingredient to understand a number of physical phenomena involving correlated many-particle systems such as quantum phase transitions, the quantum Hall effect, and high-temperature superconductivity [10-12]. Among the various physical systems of identical particles, particular attention has been devoted to the analysis of multipartite entanglement in a noninteracting fermion gas. It has been shown that the multiparticle entanglement can be built only out of two-fermion entanglement [13]. The existence and the nature of the entanglement in systems of three noninteracting fermions have also been analyzed by means of different parametrized entanglement witnesses [14-16] that enable the detection of two different classes of tripartite entangled states, namely Greenberger-Horne-Zeilinger (GHZ) and $W$ states. By using the Zanardi approach [3], that is, by mapping the Fock space of a system of fermions into the isomorphic mode state, a geometric measure of entanglement for $N$ fermions with spin $\frac{1}{2}$ has also been proposed [17]. A general entanglement criterion applicable to both bosonic and fermionic systems has recently been introduced though it is defined only with reference to the measurement setup [5].

In this work, we discuss an entanglement criterion suitable both for three-fermion and for three-boson mixed states. We follow the basic concepts of the method proposed by Wiseman and Vaccaro [4] for the analysis of bipartite entanglement in systems of identical particles. Our criterion does not violate the particle local number superselection rule. In fact here the entanglement between three subsets, say $A, B, C$, is given by the average, over the local particle number, of the number of quantum correlations existing among the standard quantum registers of three subsets each with a 
definite local particle number. Given the use of the concept of standard quantum registers, the tripartite negativity (TPN), recently introduced to quantify the tripartite entanglement of nonidentical subsystems [18,19], is here taken as a measure of the entanglement exhibited by three-boson or three-fermion mixed states. In order to get a better understanding of the criterion, first we compare and contrast it to other approaches, evaluating the quantum correlations in many-particle systems. Then we apply it to investigate the entanglement dynamics in a simple model mimicking the continuous-time quantum walks (QWs) [20] of three identical particles (bosons or fermions) in a one-dimensional structure. Specifically, QWs of indistinguishable elements constitute the ideal systems to test and validate our theoretical approach since in them the exchange terms lead to the formation of correlations between the particles even in absence of interaction, as shown by the experimental investigations on two-photon propagation in lattices of coupled waveguides [21,22].

The paper is organized as follows: In Sec. II we define the tripartite entanglement criterion in boson and fermion systems with reference to other entanglement measures in systems of identical particles. The main properties of the TPN as a measure of the number of quantum correlations in a six-mode system and its comparison with the geometric measure introduced in Ref. [17] are discussed in Sec. III. In Sec. IV we evaluate the time evolution of the TPN in QWs of three particles propagating in a lattice for two different cases: particles following the Bose-Einstein statistics and particles following the Fermi-Dirac statistics. Finally, we present our conclusions in Sec. V.

\section{TRIPARTITE ENTANGLEMENT CRITERION}

In this section, we introduce an entanglement criterion for boson and fermion systems relying on the concept of entanglement of particles [4].

In order to represent the mixed quantum state of $N$ identical particles, we adopt the occupation-number representation. Thus, in a system with $L$ modes an arbitrary mixed state of $N$ bosons (fermions) can be written as [23]

$$
\rho=\sum_{\{n\},\left\{n^{\prime}\right\}} f\left(\{n\},\left\{n^{\prime}\right\}\right)|\{n\}\rangle\left\langle\left\{n^{\prime}\right\}\right|,
$$

with the integers $n_{i}\left(n_{i}^{\prime}\right)$ of the set $\{n\}=n_{1}, \ldots, n_{i}, \ldots, n_{L}$ $\left(\left\{n^{\prime}\right\}=n_{1}^{\prime}, \ldots, n_{i}^{\prime}, \ldots, n_{L}^{\prime}\right)$ satisfying $n_{1}+\cdots+n_{i}+\cdots+$ $n_{L}=N\left(n_{1}^{\prime}+\cdots+n_{i}^{\prime}+\cdots+n_{L}^{\prime}=N\right)$. The ket $|\{n\}\rangle$ is the state of the Fock space with $n_{i}$ particles in the singleparticle modes $i$, and $f\left(\{n\},\left\{n^{\prime}\right\}\right)$ are the coefficients in the superposition. While for bosons $n_{i}$ ranges from 0 to $N$, for fermions the occupation numbers are restricted to 0 and 1 by the Pauli exclusion principle.

A formal equivalence between the Fock space and the space obtained as a tensor product of $L$-dimensional singleparticle subspaces can be established [3]. In such a procedure, the occupation number of each mode represents a distinct state of the mode itself. Zanardi and Lari et al. [3,17] defined as entanglement of a system of identical particles the so-called entanglement of modes $\left(E_{M}\right)$, namely the number of quantum correlations appearing among the occupation numbers of the modes.
As argued by some authors [24,25], an application of the $E_{M}$, as it is, to the quantum states of many-body systems could lead to misleading results since such a measure quantifies the entanglement between modes and not between the particles. In order to clarify this point, let us consider a single particle in an equal superposition of three modes, so that in the occupationnumber representation its state $|\chi\rangle$ reads

$$
|\chi\rangle=\frac{1}{\sqrt{3}}(|001\rangle+|010\rangle+|100\rangle) .
$$

By using the geometric measure of the entanglement relying on the isomorphism between the Fock space and the mode space [17], we can evaluate the tripartite entanglement $\epsilon_{G}$ between the modes as

$$
\epsilon_{G}=\|\tau\|-\|\tau\|_{\mathrm{sep}}
$$

where

$$
\|\tau\|=\sqrt{\sum_{i, j, k}^{3}\left|\left\langle\chi\left|\sigma_{i} \otimes \sigma_{j} \otimes \sigma_{k}\right| \chi\right\rangle\right|^{2}} \quad \text { and } \quad\|\tau\|_{\text {sep }}=1
$$

with $\left\{\sigma_{i}\right\}$ the set of three generators of the $\mathrm{SU}(2)$ group, namely the Pauli operators, each acting on a single-mode space. After straightforward calculations, one finds that the tripartite entanglement of $|\chi\rangle$ is different from 0 , specifically $\epsilon_{G}=\sqrt{33} / 3-1$. Such a result would seem to indicate a single-particle nonlocality stemming from the entanglement with the vacuum [24,25]. However, the above entanglement is not apparent when the particle wave function $\chi(x)$ is considered in the space configuration

$$
\chi(x)=\frac{1}{\sqrt{3}}\left[\psi_{1}(x)+\psi_{2}(x)+\psi_{3}(x)\right],
$$

with $\psi_{i}$ indicating the single-particle states.

To explain the emerging paradox, the appearance of nonlocality has been related to multiparticle effects [25]. Anyway, this behavior raises some questions on the use of the criteria relying on $E_{M}$, since they do not always capture the true entanglement between the parties of the system. As argued by Wiseman and Vaccaro [4], in particular such criteria fail to take into account the local particle number (LPN) superselection rule. In fact each party of the system must be able to perform arbitrary local operations on its modes in order to fully use the entanglement between the modes. Unless each subsystem possesses a definite number of particles, such local operations violate the superselection rule for the LPN and are not possible in practice. Thus in general, the entanglement measures relying on $E_{M}$ overestimate the available entanglement.

A tripartite entanglement criterion obeying the local particle number superselection rule can be obtained by extending the operational definition of bipartite entanglement of identical particles introduced by Wiseman and Vaccaro [4,6]. Being Alice, Bob, and Charlie, the three parties of a quantum system in the mixed state $\rho$ each accessing a given set of modes, here we assume that any subsystem possesses a standard quantum register, namely a set of distinguishable qubits in addition to the indistinguishable particles described by $\rho$. We define as tripartite entanglement $\epsilon_{T}$ the maximum number of quantum correlations that Alice, Bob, and Charlie can produce between 
their standard quantum registers by means of local operations. As a consequence of the LPN superselection rule, this tripartite entanglement in place of quantum correlations between the modes that Alice, Bob, and Charlie have access is given by

$$
\epsilon_{T}=\sum_{n_{A}, n_{B}, n_{C}}^{n_{A}+n_{B}+n_{C}=N} P_{n_{A}, n_{B}, n_{C}} \epsilon_{A B C}\left(\rho_{n_{A}, n_{B}, n_{C}}\right),
$$

where $\rho_{n_{A}, n_{B}, n_{C}}=\Pi_{n_{A}, n_{B}, n_{C}} \rho \Pi_{n_{A}, n_{B}, n_{C}}$ is obtained from $\rho$ by means of the projectors $\Pi_{n_{A}, n_{B}, n_{C}}$ onto fixed LPN states ( $n_{A}$ for Alice, $n_{B}$ for Bob, and $n_{C}$ for Charlie). $P_{n_{A}, n_{B}, n_{C}}=$ $\operatorname{Tr}\left(\rho_{n_{A}, n_{B}, n_{C}}\right)$ is the probability of finding $n_{A}, n_{B}$, and $n_{C}$ as a result of a measurement of the local particle number by Alice, Bob, and Charlie, respectively, while $\epsilon_{A B C}$ is an entanglement standard measure which quantifies the degree of tripartite entanglement among the three sets of modes each controlled by a party of the system. It is worth noting that here is used a standard measure of the tripartite entanglement, that is, a measure of the tripartite entanglement of nonidentical subsystems, since the standard quantum registers of Alice, Bob, and Charlie consist of distinguishable qubits. Specifically, since some good measures $\epsilon_{A B}$ of bipartite entanglement can successfully be extended to multipartite systems by considering bipartite partitions of them [26], we take as standard tripartite entanglement measure the geometric mean of the entanglement measures of the bipartitions of the system:

$$
\epsilon_{A B C}=\sqrt[3]{\epsilon_{A-B C} \epsilon_{B-A C} \epsilon_{C-A B}}
$$

in agreement with that proposed in the literature [18,19,27]. Thus, expression (6) now reads

$$
\epsilon_{T}=\sum_{n_{A}, n_{B}, n_{C}}^{n_{A}+n_{B}+n_{C}=N} P_{n_{A}, n_{B}, n_{C}} \sqrt[3]{\epsilon_{A-B C}\left(\rho_{n_{A}, n_{B}, n_{C}}\right) \epsilon_{B-A C}\left(\rho_{n_{A}, n_{B}, n_{C}}\right) \epsilon_{C-A B}\left(\rho_{n_{A}, n_{B}, n_{C}}\right)} .
$$

The tripartite entanglement $\epsilon_{T}$ does not violate the LPN superselection rule. In fact when Alice, Bob, and Charlie measure their local particle numbers $n_{A}, n_{B}$, and $n_{C}$, the mixed state $\rho$ collapses, with a given probability $P_{n_{A}, n_{B}, n_{C}}$, in $\rho_{n_{A}, n_{B}, n_{C}}$. Local operations can be performed on the latter without any restrictions in order to transfer its entanglement $\epsilon\left(\rho_{n_{A}, n_{B}, n_{C}}\right)$ to the standard quantum registers of each subsystem. Since $\epsilon_{T}$ is the weighted sum of the terms $\left[\epsilon_{A-B C}\left(\rho_{n_{A}, n_{B}, n_{C}}\right) \epsilon_{B-A C}\left(\rho_{n_{A}, n_{B}, n_{C}}\right) \epsilon_{C-A B}\left(\rho_{n_{A}, n_{B}, n_{C}}\right)\right]^{1 / 3}$, the entanglement is not affected, on the average, by measurements on the local particle number.

Unlike $\epsilon_{G}$, the tripartite entanglement of the single-photon state written in Eq. (2) according to our criterion is 0. By considering the partition of the system in three subsystems each controlling a single-mode state, the state $|\chi\rangle$ is given by the linear superposition of three quantum states having different LPNs and therefore the contribution to $\epsilon_{T}$ from each term is zero. This result again supports the interpretation of $\epsilon_{T}$ as a measure of the tripartite entanglement not among the modes but among the particles of the systems, in agreement with the analogous investigations performed for the bipartite case $[4,6]$.

\section{TPN IN A SIX-MODE SYSTEM}

In order to obtain $\epsilon_{T}$ according to the criterion given in Eq. (8), the evaluation of the bipartite standard entanglement $\epsilon_{I-J K}\left(\rho_{n_{A}, n_{B}, n_{C}}\right)$ for all bipartitions $I-J K$ of each possible set of LPNs is needed (with $I=A, B, C$ and $J K=B C, A C, A B$ ). Unfortunately, the latter is a very challenging task in highdimensional systems for mixed states of multiparticle systems.

In this section, we intend to give a nonambiguous measure of the tripartite entanglement $\epsilon_{T}$ which can be used in a practical way in a simple system and then compare it with the geometric measure [17]. To this aim, we restrict our analysis to the case of three bosons (fermions) each in a six-mode single-particle space $h_{6}$. The Fock space $H_{6}(3)$ of the full system is the totally symmetric (antisymmetric) subspace of the tensor product $h_{6}^{\otimes 3}$. By partitioning the system in three subsets, each acceding two modes, we note that only a few quantum states belonging to the Fock space $H_{6}(3)$ give a nonvanishing contribution to the tripartite entanglement according to the criterion given in Eq. (8). Let us examine the case of the set of mixed states $F_{\left(n_{A}=0\right)}:=\operatorname{span}\left\{\rho_{\left(0, n_{B}, n_{C}\right)}\right\}$ $\left(n_{B}, n_{C}=0,1,2,3\right.$ with $\left.n_{B}+n_{C}=3\right)$ where the local number of particles possessed by Alice is zero. Any state of $F_{\left(n_{A}=0\right)}$ can be written as $|00\rangle\left\langle\left. 00\right|_{A} \otimes \rho_{B C}\right.$ and therefore it is biseparable; that is, it can be factorized in a term describing Alice with no particle and in a term describing the Bob and Charlie subsets with three particles. The bipartite entanglement relative to the bipartition $A-B C$ is zero and, in turn, $\epsilon_{T}$ is vanishing according to Eq. (8). This implies that the entanglement due to sets of quantum states with a LPN equal to zero is vanishing. On the other hand, the tripartite number of quantum correlations can be different from zero only when each party has one particle, that is, only for the quantum states belonging to the set $F_{\left(n_{a}=1, n_{b}=1, n_{c}=1\right)}:=\operatorname{span}\left\{\rho_{(1,1,1)}\right\}$. Since each partition has two modes, the subspace spanned by the vectors of $F_{\left(n_{a}=1, n_{b}=1, n_{c}=1\right)}$ turns out to be isomorphous to the three-qubit Hilbert space $\mathbb{C}^{2} \otimes \mathbb{C}^{2} \otimes \mathbb{C}^{2}$. Thus the entanglement of three identical particles (both bosons and fermions) in this case can be evaluated in terms of the quantum correlations between three qubits.

Different approaches have been developed to characterize the tripartite entanglement in system of distinguishable qubits. The three-tangle or residual entanglement related to squares of the bipartite concurrences has been proposed [28], even if its use is questioned since it does not properly quantify the threeparty entanglement for $W$ states [29]. Alternatively, as stated in the previous section, standard tripartite entanglement can be estimated by means of some good measures of the number of quantum correlations in bipartite systems whose Hilbert space 
is $\mathbb{C}^{2} \otimes \mathbb{C}^{4}$. It should be noticed that in this case both von Neumann entropy and concurrence present weaknesses [18]. The former is an appropriate measure only for pure states; the latter, even if it is well defined for mixture states of two qubits, is applicable to higher dimensions only for pure states. A valid measure of tripartite entanglement for nonpure states can be obtained by using the negativity. From the Eq. (7), the tripartite negativity (TPN) $\mathcal{N}_{A B C}$ can be defined as

$$
\mathcal{N}_{A B C}=\sqrt[3]{\mathcal{N}_{A-B C} \mathcal{N}_{B-A C} \mathcal{N}_{C-A B}}
$$

where the bipartite negativities are given by $N_{I-J K}=$ $\sum_{i}\left|\gamma_{i}\left(\rho^{T_{I}}\right)\right|-1$ with $\gamma_{i}\left(\rho^{T_{I}}\right)$ the eigenvalues of $\rho^{T_{I}}$ which is the partial transpose related to the subsystem $I$ of the total density matrix.

By inserting the TPN of Eq. (9) in Eq. (8), the tripartite entanglement of a mixed state $\rho$ of three bosons (fermions) in a six-mode system can be easily evaluated from

$\epsilon_{T}=P_{1,1,1} \sqrt[3]{\mathcal{N}_{A-B C}\left(\rho_{1,1,1}\right) \mathcal{N}_{B-A C}\left(\rho_{1,1,1}\right) \mathcal{N}_{C-A B}\left(\rho_{1,1,1}\right)}$,

once we know the projection of $\rho$ on the subspace where the local number of each party is fixed to 1 , namely $\rho_{1,1,1}=$ $\Pi_{1,1,1} \rho \Pi_{1,1,1}$.

In order to illustrate some features of the entanglement measure introduced in Eq. (10) in comparison with the geometric measure $\epsilon_{G}$, here we consider a six-mode threefermion system in the state

$$
\begin{aligned}
|\Phi\rangle= & \cos \alpha \cos \beta|010101\rangle+\cos \alpha \sin \beta|101010\rangle \\
& +\frac{\sin \alpha}{\sqrt{2}}|111000\rangle+\frac{\sin \alpha}{\sqrt{2}}|000111\rangle,
\end{aligned}
$$

where $\alpha, \beta$ are phases ranging from 0 to $\pi$. If we consider the following partition of the system $A=\{1,2\}, B=\{3,4\}$, and $C=\{5,6\}$, we note that $|\Phi\rangle$ is a linear superposition of states, each with a given LPN for any set of modes. When $\alpha=0, \pi$ and $\beta=(\pi / 4),(3 \pi / 4)$, only the first two terms are nonvanishing and a GHZ state with each party of the system containing a particle, namely, $n_{A}=n_{B}=n_{C}=1$, is obtained. On the other hand for $\alpha=(\pi / 2)$, a specific LPN cannot be ascribed to the subsystems. Our goal is to evaluate the number of quantum correlations of $|\Phi\rangle$ according to $\epsilon_{T}$ and $\epsilon_{G}$. The latter can be calculated again from Eq. (3), where now [17]

$$
\begin{aligned}
\|\tau\| & =\sqrt{\sum_{i, j, k=1}^{15} 8\left|\left\langle\Phi\left|\lambda_{i} \otimes \lambda_{j} \otimes \lambda_{k}\right| \Phi\right\rangle\right|^{2}} \quad \text { and } \\
\|\tau\|_{\text {sep }} & =6 \sqrt{6}
\end{aligned}
$$

with $\left\{\lambda_{i}\right\}$ indicating the set of fifteen generators of the SU(4) group [30] acting on the two sites of one subset.

Figure 1 displays $\epsilon_{T}$ and $\epsilon_{G}$ as a function of $\alpha$ and $\beta$. The two entanglement measures show a different behavior, and in agreement with the theoretical predictions of the previous section the geometric measure is always greater than the TPN. The latter is vanishing for any value of $\beta$ when $\alpha=\pi / 2$; in this case, $|\Phi\rangle$ becomes the linear superposition of two terms, each corresponding to different LPNs, thus yielding zero entanglement. At $\alpha=0, \pi$ and $\beta=(\pi / 4),(3 \pi / 4), \epsilon_{T}$ exhibits four peaks indicating the maximum number of quantum correlation in the GHZ states $(1 / \sqrt{2})( \pm|010101\rangle \pm|101010\rangle)$
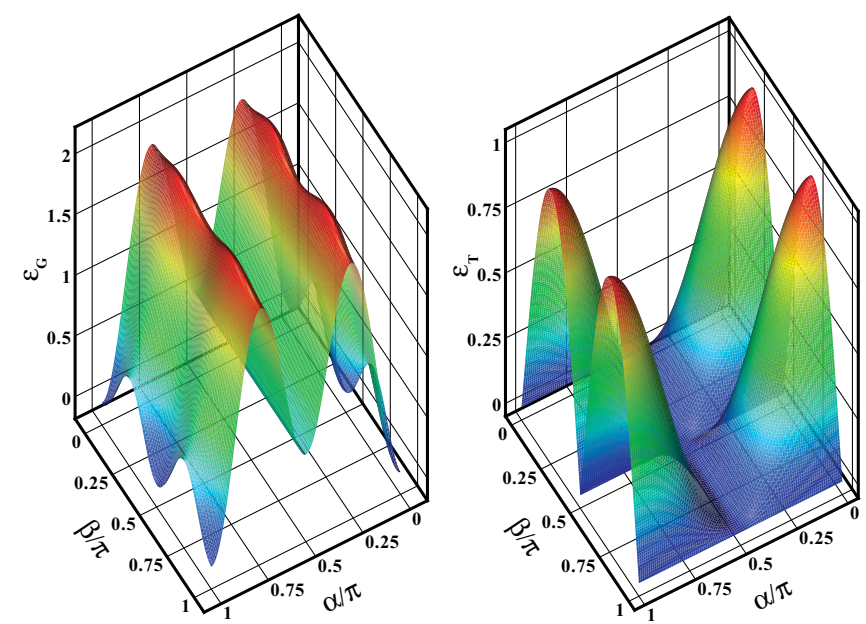

FIG. 1. (Color online) The tripartite entanglement $\epsilon_{G}$ (left panel) and $\epsilon_{T}$ (right panel) as a function of the phases $\alpha$ and $\beta$ ranging from 0 to $\pi$. Specifically, the quantum correlations exhibited by the three-fermion state $|\Phi\rangle$ of Eq. (11) among the three parties $A=\{1,2\}$, $B=\{3,4\}$, and $C=\{5,6\}$ are here considered.

describing one particle in each partition. On the other hand, the geometric measure is zero only for those values of the phases reducing the linear superposition of the four states in Eq. (11) to a single term. For $\alpha=\pi / 2, \epsilon_{G}$ shows a local minimum but unlike $\epsilon_{T}$ it is not vanishing, thus indicating a degree of nonseparability different from zero. Its maximum values are found when $\beta=(\pi / 4),(3 \pi / 4)$ and $\alpha=$ $(\pi / 4),(3 \pi / 4)$, namely when all the moduli of the coefficients of the linear superposition of Eq. (11) become equal and $|\Phi\rangle$ reduces to $\frac{1}{2}(|010101\rangle \pm|101010\rangle \pm|111000\rangle \pm|000111\rangle)$. The disagreement between the qualitative behavior exhibited by the tripartite negativity and the geometric measure for this specific case is representative of the discrepancy between the two entanglement notions. In fact, $\epsilon_{G}$ quantifies the number of quantum correlations between the modes regardless of the number of particles in any subset, while $\epsilon_{T}$ measures the entanglement of the particles in the modes.

\section{ENTANGLEMENT DYNAMICS IN THREE-BOSON AND THREE-FERMION QUANTUM WALKS}

In the last years, theoretical and experimental investigations have shown how the quantum statistics, due to exchange symmetry, may play a key role in the appearance of quantum correlations between noninteracting identical particles in various systems, ranging from the propagation of photons [31] to electron transport in the integer quantum Hall effect [32]. Among the different physical phenomena examined, the continuous-time quantum walks (QWs) have received great interest since they represent an ideal laboratory to observe many-particle quantum mechanical behavior and to implement future quantum technologies [21,22,33,34]. Specifically, the emergence of nonclassical correlations in QWs of two photons in an array of coupled waveguides has experimentally been observed [21,22]. By using the criterion based on TPN described in the previous section, here we investigate the entanglement dynamics of the continuous-time QWs of three bosons and of three fermions in an array of six sites. 
In order to study the three-particle entanglement dynamics, we follow the formalism developed in Ref. [33] for the QWs of nonclassical light in an array of coupled waveguides. We consider a one-dimensional tight-binding model, within the approximation of nearest-neighbor interaction, described by the Hamiltonian $\mathcal{H}$ :

$$
\mathcal{H}=G \sum_{i=1}^{6} c_{i}^{\dagger} c_{i}+T \sum_{i=1}^{5}\left(c_{i}^{\dagger} c_{i+1}+c_{i+1}^{\dagger} c_{i}\right),
$$

where $c_{i}^{\dagger}\left(c_{i}\right)$ is the creation (annihilation) operator for a particle in the $i$ th site which obeys the commutation relation $\left[c_{i}, c_{j}^{\dagger}\right]_{ \pm}=\delta_{i j}$, where the upper sign holds for bosons and the lower one for fermions [35]. $G$ is the on-site energy and $T$ is the tunneling rate at which the particles are transferred to the neighboring sites. Here we are considering a lattice with reflecting boundary conditions, so that the transition amplitude from the node 1 (6) to the node 6 (1) is zero. As will be shown in the following, this implies that the time evolution of the quantum system is not periodic, unlike the QWs of particles in lattices with periodic boundary conditions [36,37].

By diagonalizing the Hamiltonian (13), in the Heisenberg picture the annihilation operator $c_{r}$ at time $t$ can be obtained as [33]

$$
\begin{aligned}
c_{r}(t)= & \sum_{s=1}^{6} c_{s}(0) C_{r s}, \\
C_{r s}= & \frac{2}{7} \exp \left(-\frac{i G t}{\hbar}\right) \sum_{k=1}^{6} \exp \left[-\frac{2 i t T}{\hbar} \cos \left(\frac{k \pi}{7}\right)\right] \\
& \times \sin \left(\frac{r k \pi}{7}\right) \sin \left(\frac{s k \pi}{7}\right),
\end{aligned}
$$

where $\frac{2}{7} \sum_{k=1}^{6} \sin \left(\frac{r k \pi}{7}\right) \sin \left(\frac{s k \pi}{7}\right)=\delta_{r s}$. Since any input Fock state can be expressed by means of the creation operators $c_{r}^{\dagger}$ and the vacuum state $|0,0,0,0,0,0\rangle$, its time evolution during the QWs can be calculated by using Eq. (14). Even if the effect of different initial occupation numbers on the entanglement dynamics could be considered, here, for simplicity, we only examine the case of three particles (bosons or fermions) initially coupled to three neighboring sites, i.e., the state

$$
|\Psi(t=0)\rangle=|1,1,1,0,0,0\rangle .
$$

By means of the time evolution of the quantum state $|\Psi(t)\rangle$, we can quantify the tripartite entanglement dynamics of the system by using the TPN defined in Eq. (10). To this purpose, a numerical approach has been used which allows one to evaluate at any $t$ from $|\Psi(t)\rangle$ the density matrix $\rho_{1,1,1}$ where the LPN of each subset is fixed to 1 and then to diagonalize its partial transposes for the evaluation of the bipartite negativities. Here we consider two different partitions of the system, namely $A=\{1,2\}, B=\{3,4\}, C=\{5,6\}$ and $A^{\prime}=\{1,4\}, B^{\prime}=\{2,5\}, C^{\prime}=\{3,6\}$.

In Fig. 2 we report the entanglement of the three-boson and the three-fermion state as a function of the "time" $\tau=t T / \hbar$ for the two above partitions. We note that in all the cases the number of quantum correlations in the tripartite system under investigation oscillates with time though such oscillations are not periodic. In fact the interference of the three-particle wave

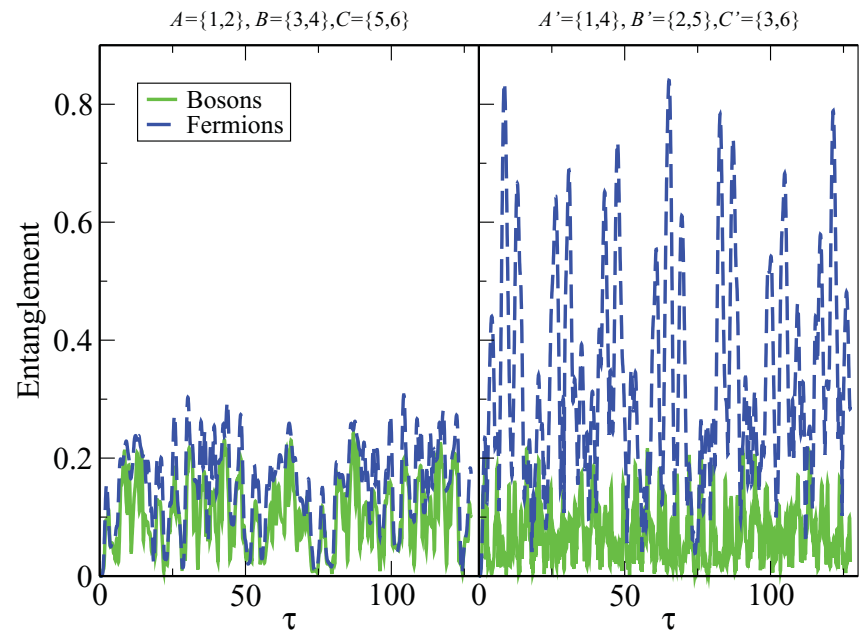

FIG. 2. (Color online) Entanglement as a function of the "time" $\tau$ for the fermionic (blue line) and bosonic (green line) systems when two different partitions are considered: $A=\{1,2\}, B=\{3,4\}$, $C=\{5,6\}$ (left panel) and $A^{\prime}=\{1,4\}, B^{\prime}=\{2,5\}, C^{\prime}=\{3,6\}$ (right panel).

function amplitudes, due to the backscattering at reflecting boundaries, results into a nonperiodic time evolution of the system. Unlike the case of single-particle dynamics in QWs into periodical structures [36,37], a time revival, namely the time interval needed to reconstruct the whole initial wave function, cannot be found here.

For both of the partitions, we note that the tripartite entanglement for the fermions is always greater than the one exhibited by the bosonic systems. This behavior can be explained by taking into account the quantum statistics of the particles. The Bose-Einstein particles exhibit bunching, thus making finite the probability to find more particles in the same site or in the same subset during the time evolution of the system. On the other hand, due to the exclusion Pauli principle, no more than one fermion can occupy the same mode. This implies that the term $P_{1,1,1}$ describing at any time the probability to find one particle in each subset and appearing in the definition of TPN of Eq. (10) increases moving from the boson to the fermion system, even if both of them are initially described by the same state $|\Psi(t=0)\rangle$. As a consequence, the tripartite entanglement among bosons becomes smaller than the one among fermions.

The TPN dynamics of the two partitions examined presents some differences. While the tripartite bosonic entanglement shows the same qualitative behavior, that is, oscillations within the interval ranging from 0 to 0.2 , the number of quantum correlations among fermions in the partition where subsets are composed of nonadjacent modes becomes, on the average, larger than the one found for subsystems controlling neighboring sites. In order to more deeply investigate this behavior, we focus on some single- and two-particle features of the quantum system at "time" $\tau=8.7$ where the fermionic TPN between $A^{\prime}, B^{\prime}, C^{\prime}$ shows a peak. In Fig. 3 the singleparticle density $\rho_{r}(t)=\left\langle c_{r}^{\dagger} c_{r}\right\rangle$ and the two-particle correlation $\Gamma_{r s}(t)=\left\langle c_{r}^{\dagger} c_{s}^{\dagger} c_{s} c_{r}\right\rangle$ are reported. Here we do not consider the three-particle correlation function, since the two-particle one is sufficient to understand the features of the entanglement, 


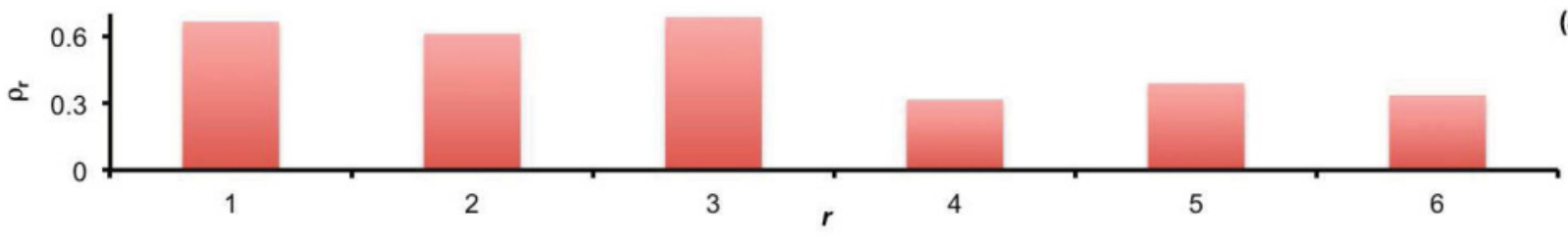

(a)

(b) Bosons

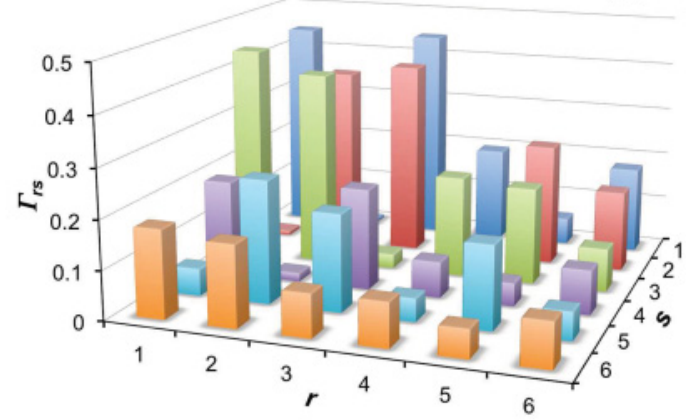

(c)Fermions

(d) Bosons
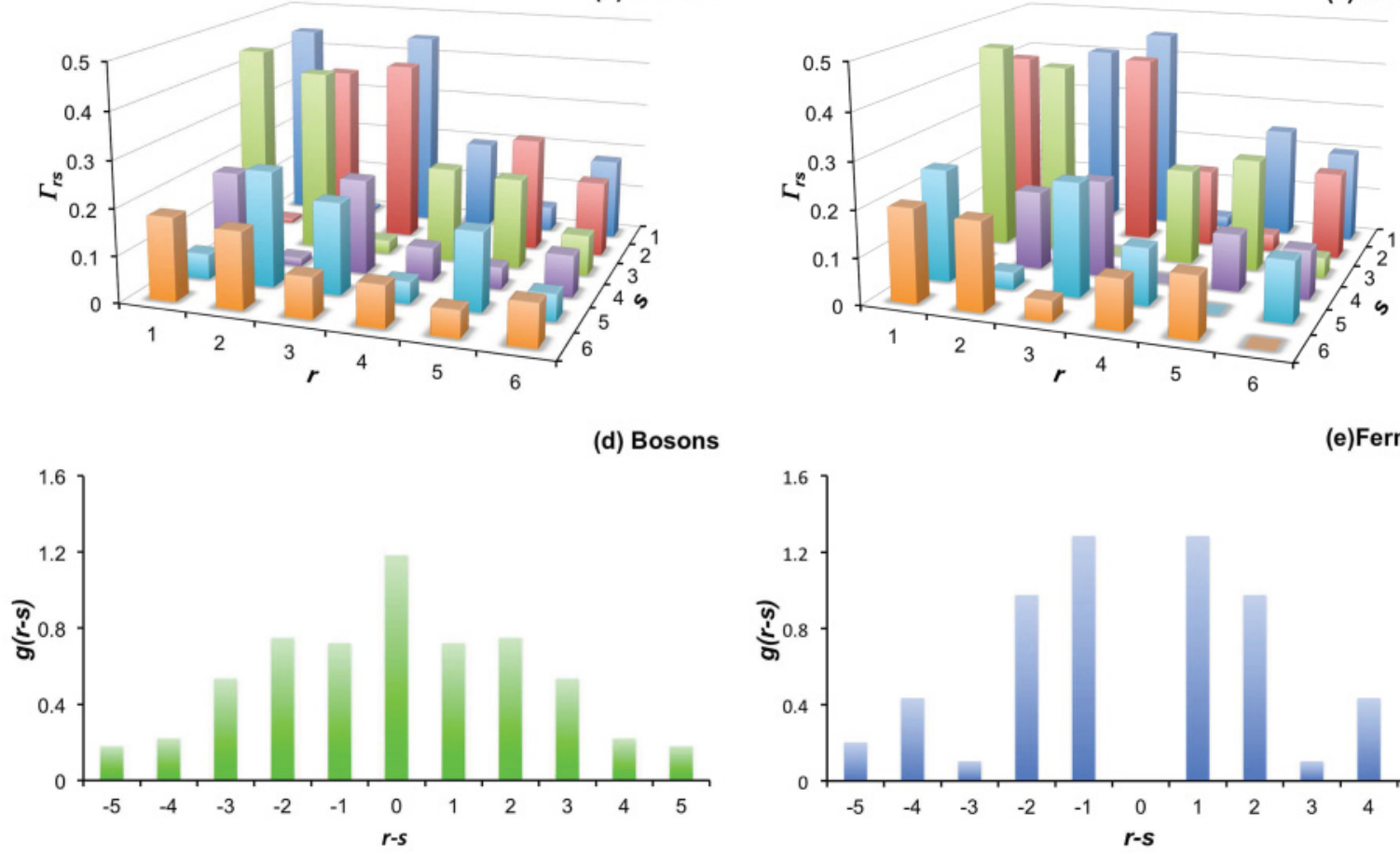

(e)Fermions

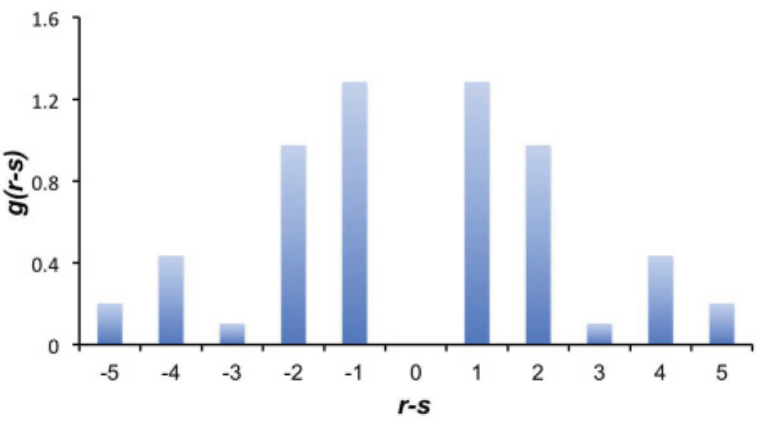

FIG. 3. (Color online) Single- and two-particle density probability of the bosonic and fermionic systems initially described by the state $|\Psi\rangle$ of Eq. (15) for $\tau=8.7$. (a) The single-particle density distribution $\rho_{r}$ which is identical for fermions and bosons. (b) The correlation matrix $\Gamma_{r s}$ for bosons computed from Eq. (16). Bosons tend to localize in the the same or different sites of the first half of the lattice. (c) The correlation matrix $\Gamma_{r s}$ for fermions computed from Eq. (17). Fermions are found with high probability in the neighboring sites of the first half of the lattice. (d) The interparticle distance probability for bosons. They tend to appear at the same site or at neighboring sites. (e) The interparticle distance probability for fermions. They are more likely to be separated by a small number (1-2) of sites.

as will be shown in the following. $\rho_{r}(t)$ does not permit us to analyze the quantum properties of the system under investigation since it shows the typical features of the classical QWs of two uncorrelated particles. As a matter of fact, the single-particle density whose expression in terms of the coefficients $C_{r s}$ reads $\rho_{r}(t)=\sum_{s=1}^{6}\left|C_{r s}\right|^{2} n_{s}$ (with $n_{s}$ the number of particles in the site $s$ at $t=0$ ) does not depend upon quantum statistics and turns out to be identical for bosons and fermions [see panel (a) of Fig. 3].

On the other hand, in analogy with the Glauber photodetection theory [38] $\Gamma_{r s}(t)$ indicates the probability to detect at time $t$ one particle at site $r$ and the other one at site $s$ and displays the two-particle quantum correlations between the locations $r$ and $s$ depending on the quantum statistics of the particles. For bosons it is given by

$$
\begin{aligned}
\Gamma_{r s}(t)= & \sum_{p=1}^{6} \sum_{q=1}^{p-1}\left|C_{r p} C_{s q}+C_{r q} C_{s p}\right|^{2} n_{p} n_{q} \\
& +\sum_{p=1}^{6}\left|C_{r p}\right|^{2}\left|C_{s p}\right|^{2} n_{p}\left(n_{p}-1\right),
\end{aligned}
$$

while for fermions it reduces to

$$
\Gamma_{r s}(t)=\sum_{p=1}^{6} \sum_{q=1}^{p-1}\left|C_{r p} C_{s q}-C_{r q} C_{s p}\right|^{2} n_{p} n_{q} .
$$

As shown by the correlation matrix in the panels (b) and (c) of Fig. 3, bosonic bunching results in a very large probability to find two particles in the same sites in the first half of the lattice. Instead, if the particles follow the Fermi-Dirac statistics, they tend to separate to neighboring sites of the first half of the lattice [see panel (c) of Fig. 3]. Regardless of the quantum statistics, this implies that for the partition $\{A, B, C\}$ the probability that more particles occupy the same subset is very high, and in turn, this means a low value of the entanglement as depicted in the left panel of Fig. 2. On the contrary, for the partition where the subsystems control nonadjacent modes, the LPN of $A^{\prime}, B^{\prime}$, and $C^{\prime}$ is more likely to be equal to 1 and therefore the TPN can reach high values (see the right panel of Fig. 2).

Also the interparticle distance probability [34] $g(\Delta)=$ $\sum_{q} \Gamma_{q, q+\Delta}$, depicted in panels (d) and (e) of Fig. 3, supports our interpretation. Apart from the behavior in $\Delta=0$, related 
to the role played by quantum statistics in determining the probability to find two particles in the same site, $g$ is high for low values of the intersite distance both for bosonic and fermionic systems, thus confirming the localization of particles in neighboring sites.

A more exhaustive discussion of the entanglement dynamics in our model undoubtedly requires further analyses also on three-particle quantum properties of the system at any time, but this goes beyond the scope of this work.

\section{CONCLUSIONS}

In summary, we have presented a criterion for the tripartite entanglement of mixed states of indistinguishable particles (both bosons and fermions). The entanglement measure proposed deviates from the other approaches recently advanced [17]. They rely on the entanglement of modes [3] but their physical significance is not always very clear as shown here by some misleading results. As a matter of fact, such measures can give values different from zero even when applied to singleparticle states which describe a particle in equal superposition of modes. In this paper, we developed a different criterion based on the notion of bipartite entanglement of particles as introduced by Wiseman and Vaccaro [4]. It provides a valid guideline to quantify the number of quantum correlations in tripartite systems on which the allowed physical operations, such as preparation, manipulation, and measurement, do not change the local number of particles contained in each subset.

Specifically, we have shown that a good entanglement measure satisfying the requirements of the theoretical criterion introduced in this paper can be obtained from the TPN recently used in the context of nonidentical particles $[18,19]$. Indeed, it constitutes a very practical tool to evaluate the number of quantum correlations appearing in three-boson or three-fermion mixture states of low-dimensional systems, that can be extracted and then placed in standard quantum registers without any violation of the local particle number superselection rule. As an application, we have considered a simple toy model of three fermions in a system with six modes and proved the reliability of the TPN in comparison with the geometric multipartite entanglement measure introduced in Ref. [17]. In agreement with theoretical predictions, we found that TPN is greater than the geometric measure. The qualitative behaviors of the two criteria as a function of the parameters of the model are quite different thus indicating their conceptual discrepancy. The geometric measure gives an estimation of the number of quantum correlations among the occupation numbers of the sites regardless of the particles. On the other hand the TPN, even if it depends upon the local particle number in the subsystems, quantifies the entanglement among the particles themselves.

Furthermore, we have used the TPN to quantify the time evolution of the quantum correlations for the continuoustime QWs of three noninteracting particles (both bosons and fermions) in a six-mode lattice, for two different partitions of the system. As expected, we find that the entanglement dynamics is not periodic due to the presence of reflecting boundary conditions in the lattice sites. Furthermore, it strongly depends upon the quantum statistics of the particle involved in the process: The bosonic bunching leads to a large probability to find more bosons in the same site and therefore in the same subset of the system. This implies a local particle number equal to zero for another of the two particles and therefore the separability of the three-particle state. On the other hand, for fermions the Pauli exclusion principle prevents occupation numbers of sites greater than one, thus making more likely to find correlated states with one particle in each subset.

Although the criterion introduced in this paper has been mainly applied to the investigation of the tripartite entanglement in some simple models, such as continuous-time QWs, it is completely general, and its application turns out to be helpful to investigate the building up of quantum correlations in both bosonic and fermionic systems. In particular, it would be of interest to extend the TPN to those physical system with large dimensionality where new and interesting results could be expected due to higher complexity of the systems themselves and to consequent richer structure of the quantum correlations.

\section{ACKNOWLEDGMENTS}

The authors would like to thank A. Bertoni and C. Jacoboni for fruitful discussions.
[1] J. Schliemann, J. I. Cirac, M. Kuś, M. Lewenstein, and D. Loss, Phys. Rev. A 64, 022303 (2001).

[2] J. R. Gittings and A. J. Fisher, Phys. Rev. A 66, 032305 (2002).

[3] P. Zanardi, Phys. Rev. A 65, 042101(R) (2002).

[4] H. M. Wiseman and J. A. Vaccaro, Phys. Rev. Lett. 91, 097902 (2003).

[5] T. Sasaki, T. Ichikawa, and I. Tsutsui, Phys. Rev. A 83, 012113 (2011).

[6] M. R. Dowling, A. C. Doherty, and H. M. Wiseman, Phys. Rev. A 73, 052323 (2006).

[7] F. Buscemi, P. Bordone, and A. Bertoni, J. Phys. Condens. Matter 21, 305303 (2009).
[8] F. Buscemi, P. Bordone, and A. Bertoni, Phys. Rev. A 73, 052312 (2006).

[9] L. Lamata and J. León, Phys. Rev. A 73, 052322 (2006).

[10] A. Osterloh, L. Amico, G. Falci, and R. Fazio, Nature (London) 416, 608 (2002).

[11] H. Li and F. D. M. Haldane, Phys. Rev. Lett. 101, 010504 (2008).

[12] V. Vedral, New J. Phys. 6, 102 (2004).

[13] C. Lunkes, C. Brukner, and V. Vedral, Phys. Rev. Lett. 95, 030503 (2005).

[14] P. Lévay and P. Vrana, Phys. Rev. A 78, 022329 (2008).

[15] T. Vértesi, Phys. Rev. A 75, 042330 (2007).

[16] H. Habibian, J. W. Clark, N. Behbood, and K. Hingerl, Phys. Rev. A 81, 032302 (2010). 
[17] B. Lari, P. Durganandini, and P. S. Joag, Phys. Rev. A 82, 062302 (2010).

[18] C. Sabín and G. García-Alcaine, Eur. Phys. J. D 48, 435 (2008).

[19] F. Anzà, B. Militello, and A. Messina, J. Phys. B 43, 205501 (2010).

[20] N. Konno, Phys. Rev. E 72, 026113 (2005).

[21] Y. Bromberg, Y. Lahini, R. Morandotti, and Y. Silberberg, Phys. Rev. Lett. 102, 253904 (2009).

[22] A. Peruzzo et al., Science 329, 1500 (2010).

[23] C. Jacoboni, Theory of Electron Transport in Semiconductors (Springer, Berlin, 2010).

[24] D. M. Greenberger, M. A. Horne, and A. Zeilenger, Quantum Interferometry II (VHC, Weinheim, 1997).

[25] D. M. Greenberger, M. A. Horne, and A. Zeilinger, Phys. Rev. Lett. 75, 2064 (1995).

[26] C. Yu and H. Song, Phys. Lett. A 373, 727 (2009).

[27] P. Love et al., Quantum Inf. Process. 6, 187 (2007).

[28] V. Coffman, J. Kundu, and W. K. Wootters, Phys. Rev. A 61, 052306 (2000).
[29] E. Jung, D. K. Park, and J. W. Son, Phys. Rev. A 80, 010301(R) (2009).

[30] M. Führinger, S. Rachel, R. Thomale, M. Greiter, and P. Schmitteckert, Ann. Phys. (NY) 17, 922 (2008).

[31] R. Hanbury Brown and R. Q. Twiss, Nature (London) 177, 27 (1956).

[32] I. Neder et al., Nature (London) 448, 333 (2007).

[33] A. Rai, G. S. Agarwal, and J. H. H. Perk, Phys. Rev. A 78, 042304 (2008).

[34] Y. Lahini, Y. Bromberg, D. N. Christodoulides, and Y. Silberberg, Phys. Rev. Lett. 105, 163905 (2010).

[35] Here we use the convention of Ref. [23]: [a,b] $]_{-}$is the usual commutator $[a, b]=a b-b a$, while $[a, b]_{+}$is the anticommutator defined as $a b+b a$.

[36] O. Mülken and A. Blumen, Phys. Rev. E 71, 036128 (2005).

[37] X.-P. Xu, Phys. Rev. E 77, 061127 (2008).

[38] R. J. Glauber, Phys. Rev. 130, 2529 (1963). 